\title{
Managing test demand by clinicians: computer assisted guidelines
}

\author{
M Peters
}

\section{Introduction}

The need to manage demands made by clinicians on the analytical resources of laboratory medicine is not new. Influencing use of laboratories by clinicians has been a consistent theme in the literature for some 20 to 30 years, early papers appearing soon after the introduction of automated analytical systems. Despite the efforts of a dedicated few, until recently misutilisation (that is, over and under use) of laboratory resources has been perceived by the majority as a chronic condition which the laboratory service must endure. Few strategies for modifying the behaviour of clinicians have been sufficiently successful to be widely adopted and the laboratory investigation of patients with similar clinical conditions continues to vary widely both within and between hospitals. However, the imperatives of the purchaser/provider environment, in which consistent standards and quality of care, clinical audit, and the efficient use of resources are all important factors, have signalled the need to re-examine this old problem.

Guidelines, usually informally communicated, detailing how patients are to be managed in a variety of clinical situations are not new. They provide the basis of most medical education and training, and provide an effective method of managing test demand. ${ }^{1}$ However, the widespread, formal introduction of guidelines for management and investigation of patients has been resisted by some, often for emotive reasons such as a perceived reduction in clinical freedom, the introduction of "robotic" medicine, and interference with training, etc., because the clinician believes he/she knows better how to treat his/her patient, or because of the effort required in drawing them up or ensuring that they are used routinely. Despite this, guidelines are now increasingly being promoted as a way of improving care by reducing unintended or unjustified variation in clinical practice and, as a consequence, of managing test demand.

There is growing evidence that a guideline based approach to laboratory investigation of patients offers laboratory medicine a practical solution to the misutilisation of resources and to management of demand, without detriment to clinical freedom or practice. Issues of terminology, design, and implementation must be addressed and some alteration in traditional ways of working may be required, but perhaps some of these are needed anyway.

\section{Background}

The Audit Commission ${ }^{2}$ supported the concept of management of demand and suggested that this requires a combination of traditional strategies together with restriction of test repertoire and the introduction of locally agreed investigation guidelines.

Whilst the formulation of locally agreed guidelines presents a considerable challenge, their implementation in a consistent way has often proved impossible. Computer assisted guidelines have now been shown to be effective in specialist units. ${ }^{3}$ Routine use of a system at the Queen Elizabeth Hospital, Birmingham, over the past two years has resulted in significant savings in medical staff time, significant reductions in the number of laboratory investigations requested, and improved continuity of management in spite of frequent changes in junior medical staff.

In this system laboratory and patient demographic data are acquired automatically from relevant feeder systems via the hospital electronic communications network. Laboratory investigations appropriate to the individual patient's clinical condition and recent pathology are proposed at terminals in ward areas. The proposed investigations reflect locally agreed guidelines which relate to defined clinical classifications which are symptom, disease, subdisease, or drug trial based. The houseman, or other clinical staff, assign patients to one or more of these classifications which predetermine the investigations required. These classifications and the investigations proposed daily by the system for each patient are reviewed by the medical staff who may update the classification or add or delete investigations or simply accept the proposed schedule. The system provides a convenient way of communicating requests to the laboratory and reviewing results as well as comprehensive feedback of laboratory usage which facilitates audit and refinement of the guidelines.

This proactive approach is currently being applied experimentally in acute general medicine and preliminary findings are encouraging.

Other forms of computer assistance are available. The system used at the Queen Elizabeth Hospital, Birmingham, can deny inappropriate investigation proposed by the user by pointing out deviation, or possible deviation, from locally agreed practice. Computer systems incorporating this type of reactive support are becoming established in hospitals in the United States where critical care plans (that is, management guidelines) are increasingly accepted as assisting the delivery of consistent standards 
of care. Personalised order sets specifying, for individual consultants or specialties, invariable care (that is, fixed tests on fixed days) for specific clinical conditions can be created within the system used at the Queen Elizabeth Hospital and also in several hospital information support systems/order communication systems already installed in the United Kingdom. Personalised order sets cannot be modified automatically to reflect current test results and are therefore restricted to managing rather simplistic guidelines. To date, computer assistance for investigation guidelines for general practice exists only in electronic text form and ultimately suffers from the same problems as printed or electronic text guidelines in the hospital environment - a lack of readership.

Computer based developments in both the United Kingdom and the United States suggest that, just as computer technology has allowed laboratory workers to cope with the clerical labour associated with routine automated analytical procedures, this same technology will also soon enable laboratory medicine to support the introduction of investigation guidelines. This will facilitate the effective management of the demand for laboratory resources.

Guidelines management systems can only be as good as the guidelines they contain, the information technology infrastructure, and laboratory service that support them. Clinical pathologists must play an active role in assisting their clinical colleagues in designing suitable guidelines and in identifying and establishing the mechanisms needed to support their routine use.

\section{Establishing guidelines for computer reinforcement}

The objective of guidelines is to describe the best clinical practice in a particular situation. That which constitutes the best practice must be agreed locally and understood by all those with direct responsibility for patient care. Importantly, in contrast to protocols, which are increasingly perceived as prescriptive with medico-legal overtones and requiring explanation of any deviation, guidelines are merely advisory, seeking to guide and educate the less experienced in appropriate management and care. Computer systems that proactively reinforce guidelines must protect clinical freedom and responsibility by facilitating alteration of the investigation schedules they propose. Similarly, reactive systems that seek to deny inappropriate investigation must finally permit any test to be ordered at any time. Commitment to guideline management of patients, however, requires systematic audit of any deviation so that they can be modified or enforced.

In the past investigation guidelines have been criticised for providing for the typical patient only, often contributing to over or under requesting by failing to recognise individual variation-an understandable limitation in design where investigation schedules have to be computed "manually". However, it is a simple mat- ter for computers to deal with complex rules or algorithms, producing variation in basic guidelines to reflect changes in a patient's clinical condition, concurrent disease, recent pathology, etc. What is much more difficult is the promotion of the understanding of the power and flexibility afforded by this facility and the organisation needed to exploit it fully. Clinical pathologists must encourage their clinical colleagues to consider in some detail numerous cases of any clinical condition for which guidelines are to be formulated. This will ensure that the resultant guidelines reflect the investigations required throughout an episode, covering a whole spectrum of severity and a number of differing pathologies. For investigation guidelines to be useful in the routine care of patients, they must specify not only which tests are required on which days (from onset of condition or on specified day of week, or both) but also under what conditions they are required-that is, the combination of test results which, for a particular clinical classification (whether disease, subdisease, or symptom based), prompts the inclusion or exclusion of specific tests. Guidelines must cover most clinical classifications routinely encountered within a specialty if computerisation is to be of real benefit.

If computer reinforced guideline management is to work effectively, laboratory results on which decisions concerning further investigation are based must be made available, in a timely fashion, in electronic form (that is, available to the guideline management system) and not communicated by telephone or on paper. The design of investigation guidelines must reflect the availability of laboratory services - for example, any restriction in "out of hours" or weekend testing should be reflected. Conversely, laboratories may wish to consider extending or modifying the routine availability of tests required for common diagnosis to simplify guidelines and streamline investigation. It is unlikely that purchasers with whom clinicians contract will be satisfied by management plans which differ significantly for patients admitted on Mondays and Saturdays.

Guideline driven investigation of patients must be feasible in the real world of hospital admissions through the Accident and Emergency departments and dispersion through numerous geographically separate (and changing) wards and clinics. Clearly, a unique patient identifier must be available and used from the patient's point of entry to medical care. Within a hospital environment, many patients admitted have first been investigated in the Accident and Emergency department, where they are often identified and investigated under a nonstandard hospital number. This is a long established practice in many hospitals, the origins of which relate to the unavailability of medical record/reception staff or the undesirability of fully registering patients who may not ultimately be admitted, or both. This practice has always caused problems in the continuity of laboratory and patient records and these problems are exacerbated in the application of computer reinforced guidelines. 
Where temporary Accident and Emergency numbers are used, they must be merged as soon as possible with the true hospital number, usually identified when the patient is admitted to a ward. Otherwise, the guideline management system will be unaware that test results already exist for the patient and tests already performed in the Accident and Emergency department may be proposed or permitted again. Hopefully, as hospital and community information technology and management infrastructures develop this problem will disappear.

Clearly, the computer system supporting investigation guidelines must be acceptable to users. It is probable, in the long term, that support for clinical guidelines will be required in some form throughout all sectors of healthcare and that appropriate functionality will be integrated within HISS and order communication systems. Where such systems are installed, with or without guideline support, the interface between the requester and the laboratory will be altered radically. Limited experience within the United Kingdom indicates that such change can be accommodated if it is carried out on a hospital wide basis after appropriate consultation and with appropriate support.

The introduction of computer reinforced investigation guidelines requires quite extensive cross-discipline cooperation and inevitably necessitates some alteration in established working practices. This calls for strong local leadership and commitment to the introduction of guideline management and the management of change involved. Difficulties in introducing relatively minor changes in the working practices of clinical teams, phlebotomists, laboratory, and Accident and Emergency reception staff can, at best, complicate progress or, at worst, lead to total failure.

The key ingredients for the successful implementation of computer based reinforcement of guideline driven investigation are identical with those identified by Sittig and Stead ${ }^{4}$ for computer based physician order entry. Although their review is inevitably restricted largely to experience in the United States, dealing briefly with the scant literature concerning "expert" or rule based systems, the critical success factors they identified are relevantthat is, "the computer system must be fast and easy to use, the user interface must behave consistently in all situations, the institution (of guideline management) must have broad and committed involvement and direction by clinicians prior to implementation, the top leadership of the organisation must be committed to the project and a group of problem solvers and users must meet regularly to work out procedural issues."

Implications for the future

It will be difficult for laboratory medicine to resist the movement, now gathering mo- mentum throughout medicine, towards guideline based management of patients. Computer technology will inevitably provide mechanisms by which such management can be reinforced. In preparation for this, and as a matter of some urgency, clinical pathologists must begin to determine, with their clinical colleagues, existing patterns of investigation of patients starting with common clinical conditions. From this, variation in physicians' requesting patterns can be examined and following discussion, optimum investigation patterns can be developed resulting in the establishment of detailed guidelines. This process, which should be at individual hospital level, is easier for specialist units, where the number of clinical conditions encountered is lower, than for acute general medicine where illness is less defined. However, both should be tackled as the intensive investigation of the former places significant demand on laboratory resources, as does patient turnover in the latter. It is probable that many hundreds of guidelines would be required to cover all possible conditions and for some conditions, the considerable effort required will not be warranted. Some 25 clinical conditions account for $50 \%$ of general medical admissions at a local district general hospital-perhaps these would be a suitable starting point.

Development at local level will enable the profession to develop a body of experience which will not only enable it to resist externally imposed guidelines but also permit comparison with other centres, perhaps leading to the formulation of a widely based consensus on the appropriate laboratory investigation of a number of clinical conditions. A nationally, regionally, or locally based audit of outcome would enable refinement of these guidelines and would facilitate education and training of medical and laboratory staff.

The availability of widely accepted guidelines together with mechanisms for their implementation, continual assessment, and refinement would mean that all patients would be investigated in a logical and predefined way, and variations in care occasioned by changing staff would be reduced. Most laboratory staff believe that a substantial proportion of the tests they perform are not essential for the adequate clinical care of patients. To effect change in this situation by altering clinicians' perceptions of appropriate testing must be a colossal contribution to healthcare.

1 Griner PF, Liptzin B. Use of the laboratory in a teaching hospital. Ann Intern Med 1971;75:157-63.

2 The Pathology Services: A Management Review. The Audit Commission, HMSO. January, 1991.

3 Mutimer D, McCauley B, Nightingale P, Ryan M, Peters $M$, Neuberger J. Computerised protocols for laboratory investigation and their effect on use of medical time and resources. F Clin Pathol 1992;45:572-4.

4 Sittig D, Stead W. Computer-based physician order entry: the state of the art. $¥ A M I A 1994 ; 1: 108-23$. 